\title{
Steiner-point free edge cutting of tetrahedral meshes with applications in fracture
}

\author{
P. Areias ${ }^{\mathrm{a}, \mathrm{c}, *}, \mathrm{~T}$. Rabczuk $^{\mathrm{b}}$ \\ a Department of Physics, University of Évora, Colégio Luís António Verney, Rua Romão Ramalho, 59, 7002-554 Évora, Portugal \\ b Institute of Structural Mechanics, Bauhaus-University Weimar, Marienstraße 15, 99423 Weimar, Germany \\ c CERIS/Instituto Superior Técnico, University of Lisbon, Portugal
}

\section{A R T I C L E I N F O}

Keywords:

3D edge-based division

Local mesh refinement

Tetrahedral cutting

$3 \mathrm{D}$ fracture

\begin{abstract}
A B S T R A C T
Realistic 3D finite strain analysis and crack propagation with tetrahedral meshes require mesh refinement/ division. In this work, we use edges to drive the division process. Mesh refinement and mesh cutting are edgebased. This approach circumvents the variable mapping procedure adopted with classical mesh adaptation algorithms. The present algorithm makes use of specific problem data (either level sets, damage variables or edge deformation) to perform the division. It is shown that global node numbers can be used to avoid the Schönhardt prisms. We therefore introduce a nodal numbering that maximizes the trapezoid quality created by each mid-edge node. As a by-product, the requirement of determination of the crack path using a crack path criterion is not required. To assess the robustness and accuracy of this algorithm, we propose 4 benchmarks. In the knee-lever example, crack slanting occurs as part of the solution. The corresponding Fortran 2003 source code is provided.
\end{abstract}

\section{Introduction}

Division of tetrahedra with applications to fracture can make use of mesh adaptivity algorithms using edge-based division. 3D adaptivity is a classical subject and is discussed in mesh generation textbooks, cf. [11]. However, several aspects, such as the general case of edge-based division and the use of global node numbering to improve the division quality were not previously addressed. In addition, applications to fracture are infrequent and make use of specific crack front cases. We here address these issues and show applications to fracture, including slanting (see, e.g. [14] for an erosion-based algorithm predicting slanting).

Several discretization-based 3D applications make use of tetrahedron division:

- Level-set based operations and mesh creation/adaptivity for large deformations, including biomechanics applications [4].

- Visualization [13,9].

- Fracture $[2,26]$.

- Surgery modeling $[18,20]$.

- Biomechanics [4].
In terms of objective for the mesh division, we focus here on the required algorithms and a number of applications. It is known that tetrahedron mesh subdivision based on edges or faces generates five distinct members of the polyhedron family: tetrahedra, square pyramids, triangular prisms (both pentahedra) and octahedra. Tetrahedrization of square pyramids and octahedra can be made compatible with neighbour elements ${ }^{1}$ for any give face-based criteria. However, triangular prisms can degenerate in the so-called Schönhardt prism, which is non-tetrahedrizable. Some Authors have been inserting nodes inside the original tetrahedron to deal with Schönhardt prisms (cf. [24]). The reason for this ad-hoc procedure is that two tasks are simultaneously being performed: mesh improvement and tetrahedrization. Of course these are equally important and here we address them separately. In terms of tetrahedron division, past work has dealt with two distinct families of methods: edge-based [25] and face-based [22] (Table 1).

Using global numbering (use the maximum node number), it is a simple matter to show that triangular prisms can be made tetrahedrizable, as will be addressed later. Prototype quality of triangles and tetrahedra (inverse relations given by P.L. George [12]) is given by:

\footnotetext{
* Corresponding author at: Department of Physics, University of Évora, Colégio Luís António Verney, Rua Romão Ramalho, 59, $7002-554$ Évora, Portugal.

E-mail address: pmaa@uevora.pt (P. Areias).

${ }^{1}$ In the sense of a face
} 
Table 1

Case selection as a function of number of marked edges and topology (neighborhood relations).

\begin{tabular}{|c|c|c|c|}
\hline Number of marked edges & Description & Case & Local relations between local element numbering and case numbering \\
\hline $\mathbf{0}$ & Single case & \#1 & Any order \\
\hline 1 & Single case & $\# 2$ & $\mathrm{~N} 1$ and $\mathrm{N} 2$ are on the marked edge \\
\hline \multirow[t]{2}{*}{2} & A node shares two marked edges & \#3 & N3 does not share a marked edge N4 shares two marked edges \\
\hline & Otherwise & $\# 4$ & N1-N2 corresponds to the smallest local marked edge \\
\hline \multirow[t]{3}{*}{3} & A node shares 3 unmarked edges & $\# \mathbf{5}$ & N3 is the corresponding node \\
\hline & A node shares three marked edges & \#6 & $\mathrm{N} 4$ is the corresponding node \\
\hline & Otherwise & $\# 7$ & $\mathrm{~N} 1$ and $\mathrm{N} 2$ have both two marked edges \\
\hline \multirow[t]{2}{*}{4} & One node contains 3 marked edges & $\# \mathbf{8}$ & N3 shares one marked edge and N4 shares three \\
\hline & Otherwise & $\# 9$ & Both N1 and N2 are on the unmarked edge \\
\hline 5 & Single case & \#10 & Both N1 and N2 are on the unmarked edge \\
\hline 6 & Single case & \#11 & Any order \\
\hline
\end{tabular}

$Q_{\text {triangle }}=\frac{\beta_{\text {triangle }} A_{\text {triangle }}}{\sum_{i=1}^{3} l_{i}^{2}}, \quad Q_{\text {triangle }} \in[0,1]$

$Q_{\text {tetrahedron }}=\frac{\beta_{\text {tetrahedron }} V_{\text {tetrahedron }}}{\sum_{i=1}^{6} l_{i}^{3}}, \quad Q_{\text {tetrahedron }} \in[0,1]$

where $\beta_{\text {triangle }}=4 \sqrt{3}$ and $\beta_{\text {tetrahedron }}=36 \sqrt{2}$. In (1)-(2) $A_{\text {triangle }}$ is the area of the triangle and $V_{\text {tetrahedron }}$ is the volume of the tetrahedron. The edge lengths are given by $l_{i}$ with $i=1,2,3$ for the triangle and $i=1, \ldots, 6$ for the tetrahedron. The evaluation of mesh quality, the corresponding arithmetic average is used. We now discuss the division of tetrahedra based on edges.

\section{Edge-based cutting with pre-ordering}

\subsection{Marking edges and calculating the crack intersection point}

To identify the corresponding edges and mark for splitting, we have several choices, according to the main goal. Using two nodes of a given edge, let them be $N 1$ and $N 2$, we have a local edge coordinate $\xi$ such as $N 1$ corresponds to $\xi=-1$ and $N 2$ to $\xi=+1$. If the surface is known from a function $\phi(x)=0$ such that $x$ are the coordinates of a given point, we use an affine relation to obtain the marked edge and corresponding local coordinate $\xi$ :

$\xi=\frac{\phi_{1}+\phi_{2}}{\phi_{1}-\phi_{2}}$

where $\phi_{1}$ and $\phi_{2}$ are the images of $\phi$ in nodes $N 1$ and $N 2$. If $\xi \in[-1,1]$, the corresponding edge is marked. We now introduce two additional strategies for edge marking. In the case of edge length, we use the following criterion for marking:

$l_{N 1 N 2} \geq \frac{f_{m}}{2}\left(l_{\max }+l_{\min }\right)$

where $l_{N 1 N 2}$ is the deformed edge length and $l_{\max }$ and $l_{\min }$ are the maximum and minimum deformed edge lengths, respectively. In addition, the typical value of the parameter $f_{m}$ adopted here is 0.75 and is introduced to avoid excessive remeshing. For the damage problem, we have (see Fig. 1):

$(1-d) L_{N 1 N 2}+d h_{\min }<f_{m} l_{N 1 N 2}$

where $d$ is the indicative damage value at the edge, $L_{N 1 N 2}$ is the undeformed edge length and $h_{\min }$ is the minimum edge size, which is considered problem data. In (5), $f_{m}$ is introduced to incorporate the effect of $l_{N 1 N 2}$ and damage in the same inequality. A typical value of $f_{m}$ is 1.5 (Table 2).

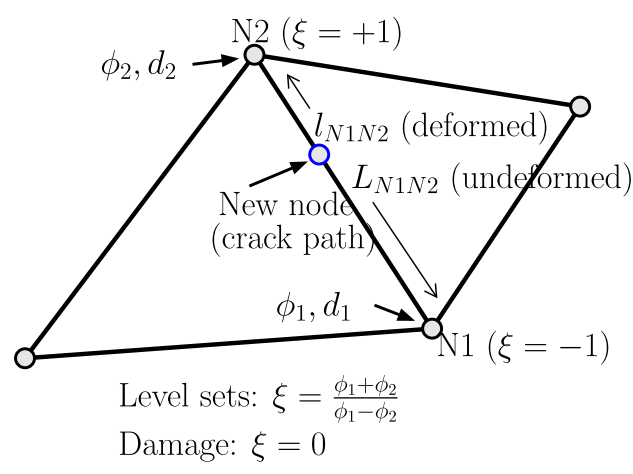

Fig. 1. Edge division based on level set or damage value.

Table 2

Index sets for tetrahedrization of \#PRI, \#PYR, \#TET and \#OCT. \#TET indicates a tetrahedron, \#PYR a pyramid, \#PRI a triangular-base prism and \#OCT a octahedron.

\begin{tabular}{ll}
\hline Solid: added edges & Indices \\
\hline \#PRI: 4-6, 1-3, 1-6 & $1,2,6,3$ \\
& $1,6,4,3$ \\
\#PRI: 4-6, 2-4, 1-6 & $1,6,5,4$ \\
& $1,2,6,4$ \\
\#PRI: 4-6, 2-4, 2-5 & $1,6,5,4$ \\
& $2,6,4,3$ \\
\#PRI: 3-5, 1-3, 1-6 & $1,2,5,4$ \\
& $2,3,6,4$ \\
\#PRI: 3-5, 1-3, 2-5 & $2,6,5,4$ \\
& $1,2,6,3$ \\
\#PRI: 3-5, 2-4, 2-5 & $1,3,5,4$ \\
& $1,6,5,3$ \\
Solid: added edges & $1,3,5,4$ \\
\#PYR: 1-3 & $2,5,1,3$ \\
\#PYR: 2-4 & $2,6,5,3$ \\
\#TET: & $1,2,5,4$ \\
\#OCT: 3-5 & $2,3,5,4$ \\
& $2,6,5,3$ \\
\#OCT: 4-6 & Indices \\
& $1,2,5,3$ \\
& $1,3,5,4$ \\
& $1,2,5,4$ \\
& $2,5,4,3$ \\
& $1,2,3,4$ \\
& $1,3,4,5$ \\
& $1,3,5,6$ \\
& $3,4,5,2$ \\
& $3,5,6,2$ \\
& $1,4,6,3$ \\
& $1,6,4,5$ \\
& $2,4,6,5$ \\
& $2,6,4,3$ \\
\hline &
\end{tabular}



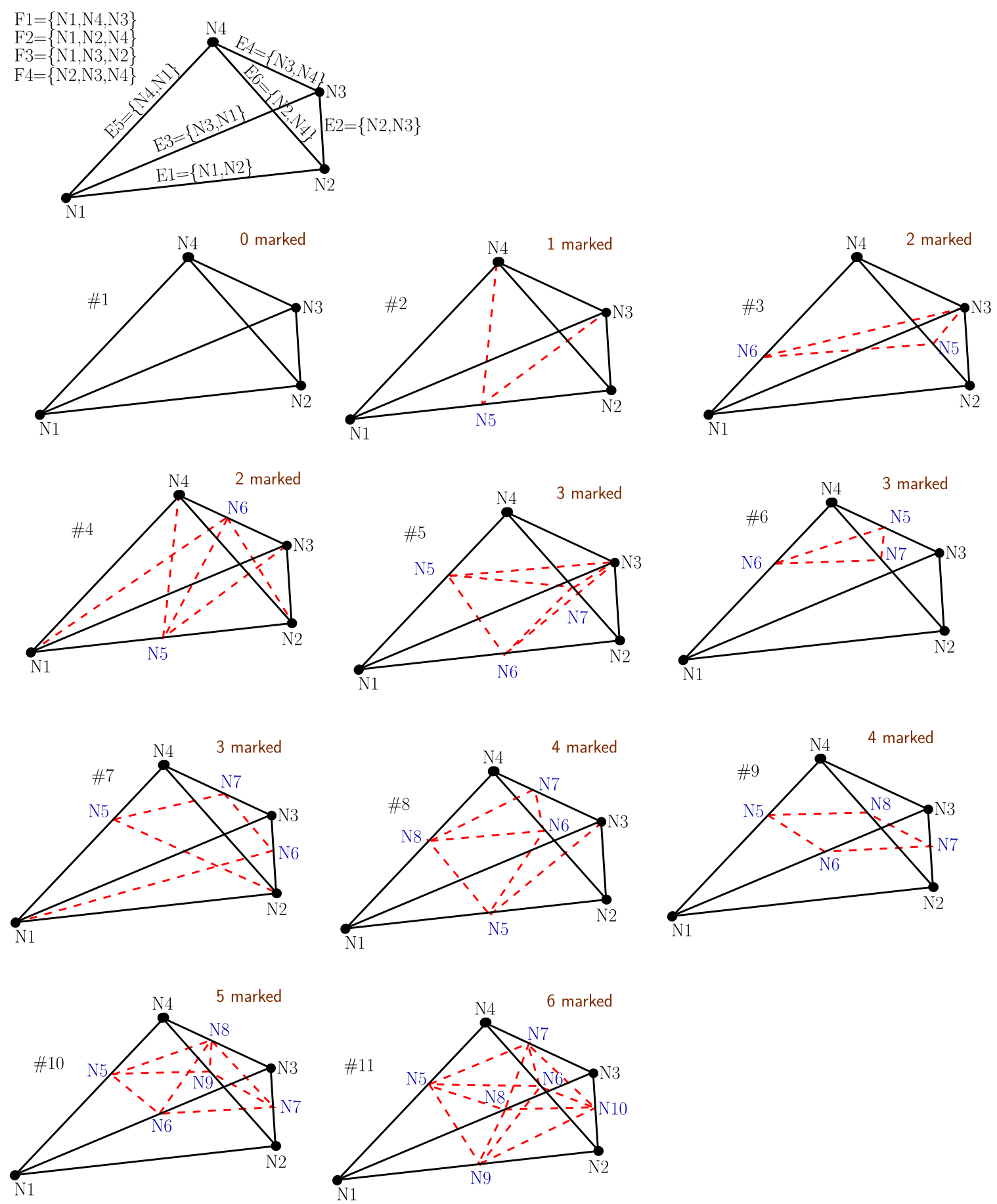

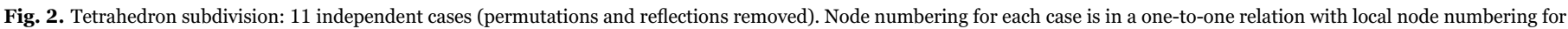
each element. 

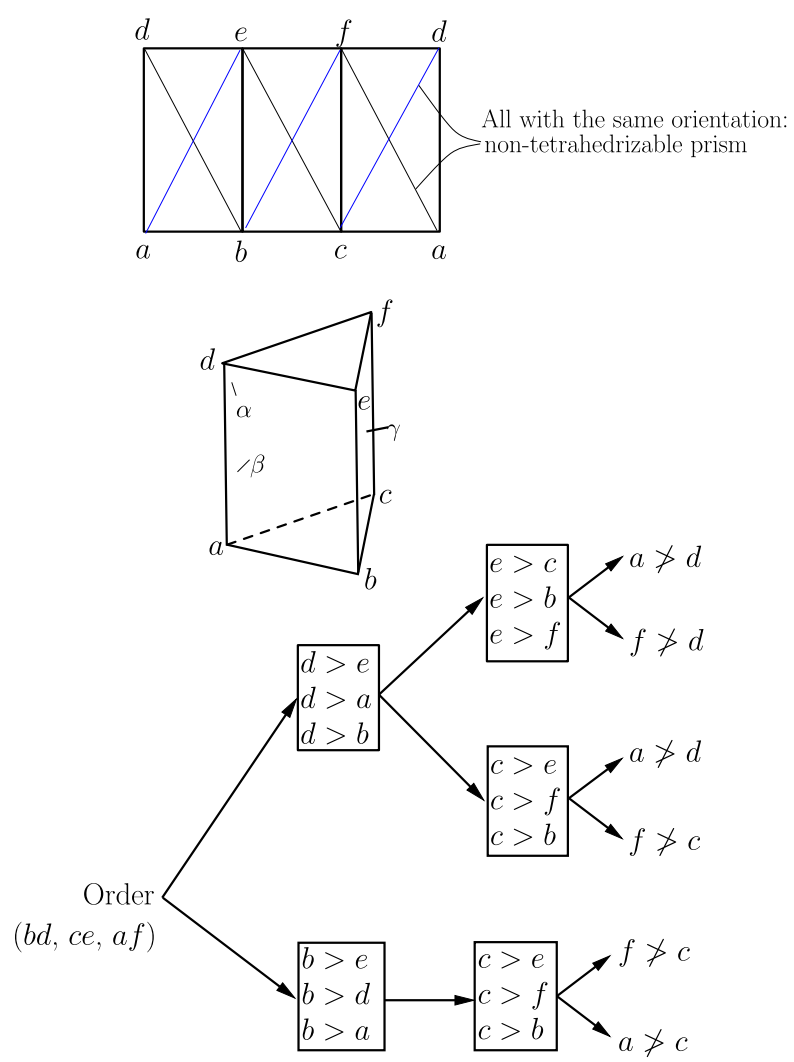

Fig. 3. Face division of a triangular prism. $a, b, c$ and $d, e$ and are global node numbers.

\subsection{Edge-based symbolic tetrahedron subdivision}

We propose a symbolic tetrahedron subdivision based on global node numbering and unequivocally split the arising quadrilateral faces. The classical 11 cases [24] of tetrahedron splitting are shown in Fig. 2. Cases where quadrilaterals appear pose a decision problem, which will be addressed later. Traditionally [24,25], the shortest dividing diagonal is used, but this can produce a non-tetrahedrizable Schönhardt prism. An alternative consists of using the global numbers as an criterion for splitting. This can produce ill-shaped elements and therefore a nodal pre-ordering is needed to ensure that elements are of satisfactory quality. Note that this process can be considered $h-$ adaptivity, see Fig. 21.6 of [8].

Since tetrahedra with adjacent faces must share the new edges, these must be compatible. The same face must be divided in a compatible form in two adjacent elements. In our method, the criterion for face bisection in two triangles is the global node number. The largest global node number in a given quadrilateral face will determine the split. It is well known that triangular prisms with divided quadrilateral faces can become non-tetrahedrizable. Fig. 3 shows a triangular prism and its planification face and the global node numbers. The sequence of largest node numbers will preclude the non-tetrahedrizable prisms (Table 3).

The division of a tetrahedron can generate the polyhedra shown in Fig. 5. It can be observed that, with the exception of the tetrahedron, division of these polyhedra is not unique and the previously mentioned criterion is adopted. Although this can result in inadequate quality (in the sense of mesh quality [8]) subsequent operations will address this aspect. Numbering of mid-edges is based on the assessment of division of trapezoids based on quality, cf. Fig. 4. For each edge $j$ we use the algorithm 1 .

Algorithm 1. Algorithm (Fortran03) for estimating edge quality.

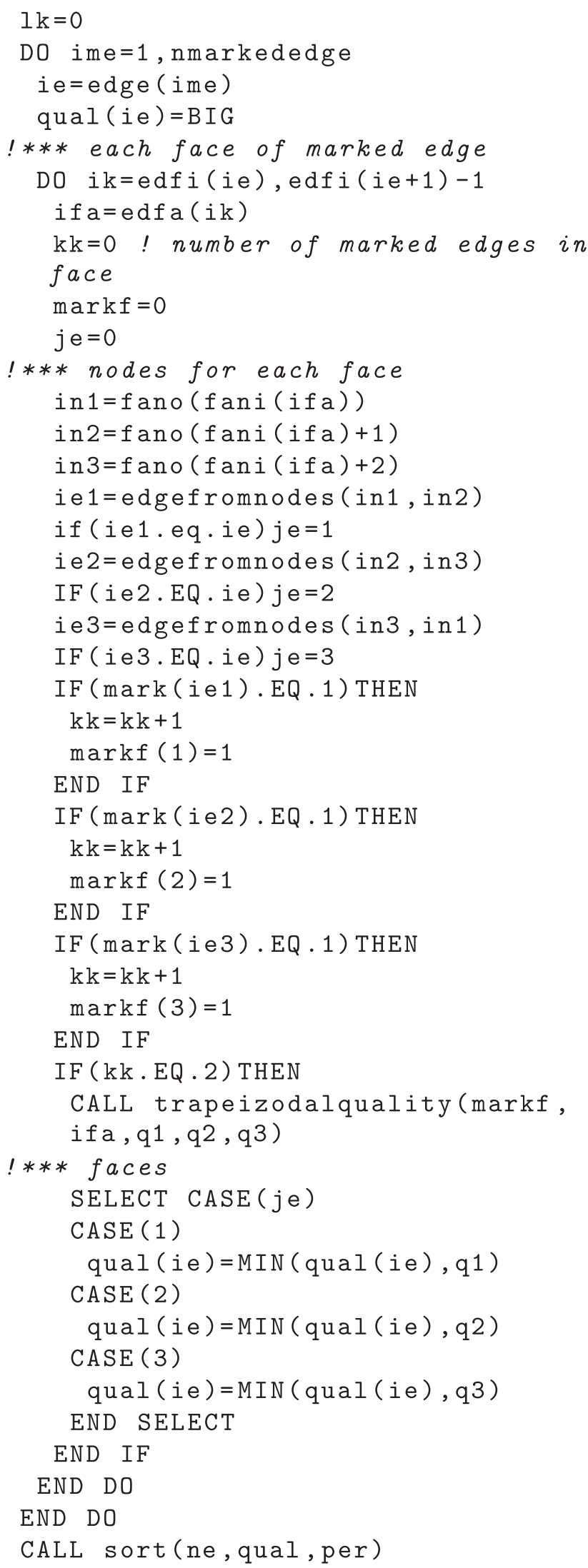

Mesh smoothing (see [8]) is performed using the centroid of incident elements see Fig. 6 and Fig.7 for the combined effects: 
Table 3

Node numbers for tetrahedrization of cases shown in Fig. 2. \#TET indicates the creation of a tetrahedron, \#PYR a pyramid, \#PRI a triangular-base prism and \#OCT a octahedron.

\begin{tabular}{|c|c|}
\hline Case & Result \\
\hline \#1 & \#TET $(1,2,3,4)$ \\
\hline \multirow[t]{2}{*}{$\# 2$} & \#TET $(3,4,5,2)$ \\
\hline & \#TET $(4,3,5,1)$ \\
\hline \multirow[t]{2}{*}{$\# \mathbf{3}$} & \#PYR(1,2,5,6,3) \\
\hline & \#TET $(6,5,3,4)$ \\
\hline \multirow[t]{4}{*}{$\# 4$} & \#TET $(2,3,5,6)$ \\
\hline & \#TET $(2,6,5,4)$ \\
\hline & \#TET $(1,5,6,4)$ \\
\hline & \#TET $(1,5,3,6)$ \\
\hline \multirow[t]{4}{*}{ \#5 } & \#TET $(2,3,6,7)$ \\
\hline & \#TET $(3,5,7,4)$ \\
\hline & \#TET $(6,3,5,7)$ \\
\hline & \#TET $(1,6,3,5)$ \\
\hline Case & Result \\
\hline \multirow[t]{2}{*}{ \#6 } & \#TET(4,7,6,5) \\
\hline & \#PRI(2,7,6,1,3,5) \\
\hline \multirow[t]{3}{*}{ \#7 } & $\# \operatorname{PYR}(6,7,4,2,5)$ \\
\hline & \#PYR(3,1,5,7,6) \\
\hline & \#TET $(1,2,6,5)$ \\
\hline \multirow[t]{4}{*}{$\# \mathbf{8}$} & \#TET $(8,6,7,4)$ \\
\hline & \#TET $(7,8,5,6)$ \\
\hline & $\# \operatorname{PYR}(2,3,7,6,5)$ \\
\hline & \#PYR(1,8,7,3,5) \\
\hline \multirow[t]{2}{*}{$\# 9$} & \#PRI $(8,7,3,4,5,6)$ \\
\hline & \#PRI(1,2,8,5,6,7) \\
\hline \multirow[t]{4}{*}{ \#10 } & \#TET $(9,8,5,4)$ \\
\hline & \#TET $(3,6,7,8)$ \\
\hline & \#PYR(6,7,9,5,8) \\
\hline & \#PRI(1,2,9,5,6,7) \\
\hline \multirow[t]{5}{*}{ \#11 } & \#TET $(1,9,8,5)$ \\
\hline & \#TET $(2,10,9,6)$ \\
\hline & \#ТET $(3,8,10,7)$ \\
\hline & \#TET $(5,6,7,4)$ \\
\hline & \#ОCT $(8,6,7,5,9,10)$ \\
\hline
\end{tabular}

$\boldsymbol{x}_{i} \leftarrow \frac{1}{\sum_{e \in \mathcal{I}_{i}} V_{e}} \sum_{e \in \mathcal{I}_{i}} \overline{\boldsymbol{x}}_{e} V_{e}$

\section{Equilibrium problems: constitutive integration in finite strains}

Using standard notation, we write the spatial equilibrium equations as [19]:

$\frac{\partial \sigma_{i j}}{\partial x_{j}}+b_{i}=0$

with the Cauchy tensor components $\sigma_{i j}(i, j=1,2,3)$. In (7) $i$ is the direction index and $j$ is the facet index. The components of the body force vector are $b_{i}$. In (7), coordinates $x_{j}$ are the spatial, or deformed, coordinates of a given point under consideration. In addition, the following natural and essential boundary conditions hold on each part of the boundary $\Gamma=\Gamma^{t} \cup \Gamma^{u}$ where $\Gamma^{t}$ is the natural boundary and $\Gamma^{u}$ is the essential boundary:

$\overline{\boldsymbol{t}}=\boldsymbol{\sigma} \cdot \boldsymbol{v} \quad$ on $\quad \Gamma^{t}$

$\overline{\boldsymbol{u}}=\boldsymbol{u} \quad$ on $\quad \Gamma^{u}$

where $\bar{t}$ is the known stress vector on $\Gamma^{t}$ where $v$ is the outer normal and $\overline{\boldsymbol{u}}$ is the known displacement field on $\Gamma^{u}$. It is assumed that (7) and (8)(9) are satisfied for a time parameter $t \in[0, T]$ with $T$ being the total time of analysis and for a point with position $x \in \Omega$ belonging to the deformed position domain at the time of analysis. Natural boundary $\Gamma^{t}$ is evolving in the sense that cracks create boundaries with known $\bar{t}$. Equilibrium configuration corresponds to the domain $\Omega$. In tensor notation, equation (7) can be presented as:

$\nabla \cdot \boldsymbol{\sigma}^{T}+\boldsymbol{b}=\mathbf{0}$

with $\nabla=\partial / \partial x$ being the spatial gradient operator. After multiplication by the velocity field $\dot{\boldsymbol{u}}$, integration in the deformed configuration $\Omega$ and application of integration by parts component-wise, we obtain the following power form $\left(\dot{W}_{\text {int }}\right.$ is the internal and $\dot{W}_{\text {ext }}$ is the external power):

$\underbrace{\int_{\Omega} \boldsymbol{\sigma}: \boldsymbol{L} \mathrm{d} \Omega}_{W_{\text {int }}}=\underbrace{\int_{\Omega} \boldsymbol{b} \cdot \dot{\boldsymbol{u}} \mathrm{d} \Omega+\int_{\Gamma^{t}} \overline{\boldsymbol{t}} \cdot \dot{\boldsymbol{u}} \mathrm{d} \Gamma}_{W_{\mathrm{ext}}}$

where $\boldsymbol{L}$ is the velocity gradient: $\boldsymbol{L}=\frac{\partial \dot{x}}{\partial x}=\frac{\partial \dot{u}}{\partial x}$.

Using the undeformed configuration $\Omega_{0}$ for the left-hand side, it is well known that (11) can be written as:

$\underbrace{\int_{\Omega_{0}} \boldsymbol{S}: \dot{\boldsymbol{E}} \mathrm{d} \Omega_{0}}_{W_{\text {int }}}=\underbrace{\int_{\Omega} \boldsymbol{b} \cdot \dot{\boldsymbol{u}} \mathrm{d} \Omega+\int_{\Gamma^{t}} \overline{\boldsymbol{t}} \cdot \dot{\boldsymbol{u}} \mathrm{d} \Gamma}_{W_{\mathrm{ext}}}$

where $S$ is the second Piola-Kirchhoff stress and $\boldsymbol{E}$ is the corresponding Green-Lagrange tensor. Eigenvalues of $\boldsymbol{E}$ are identified as $\varepsilon_{1}, \varepsilon_{2}$ and $\varepsilon_{3}$. For the quasi-brittle model employed in this work, we use the Mazars [17] definition of equivalent strain, here denoted $\varepsilon_{\mathrm{eq}}$ :

$\varepsilon_{\mathrm{eq}}=\sqrt{\sum_{i=1}^{3}\left\langle\varepsilon_{i}\right\rangle^{2}}$

with $\left\langle\varepsilon_{i}\right\rangle=\frac{\varepsilon_{i}+\left|\varepsilon_{i}\right|}{2}, i=1,2,3$. From (13) the maximum equivalent strain $\varepsilon$ is defined as $\varepsilon=\max _{\text {hist }}\left[\varepsilon_{\text {eq }}\right]$. With a non-local representation of $\varepsilon$, which is denoted $\bar{\varepsilon}$ we use a strict approach: only the softening function depends on $\bar{\varepsilon}$. Note that in the literature, $\varepsilon_{\text {eq }}$ is frequently adopted for the nonlocal strain approach, e.g. [21]. Loading/unloading conditions strictly involve local quantities and the structure of the local constitutive representation remains. Defining the equivalent stress as $\sigma_{\mathrm{eq}}=E \varepsilon_{\mathrm{eq}}$ and introducing a softening function $\bar{\sigma}(\bar{\varepsilon})$, we establish the
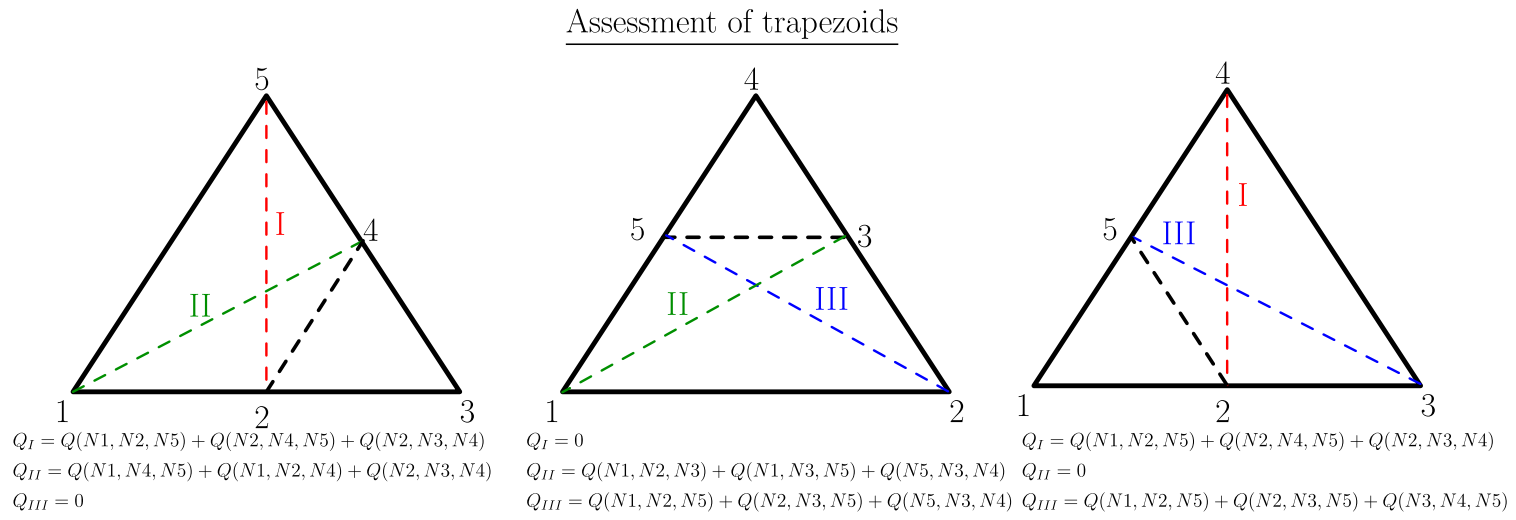

Fig. 4. Assessment of triangular (with a sub-quadrilateral) division corresponding to the face arrangement. 

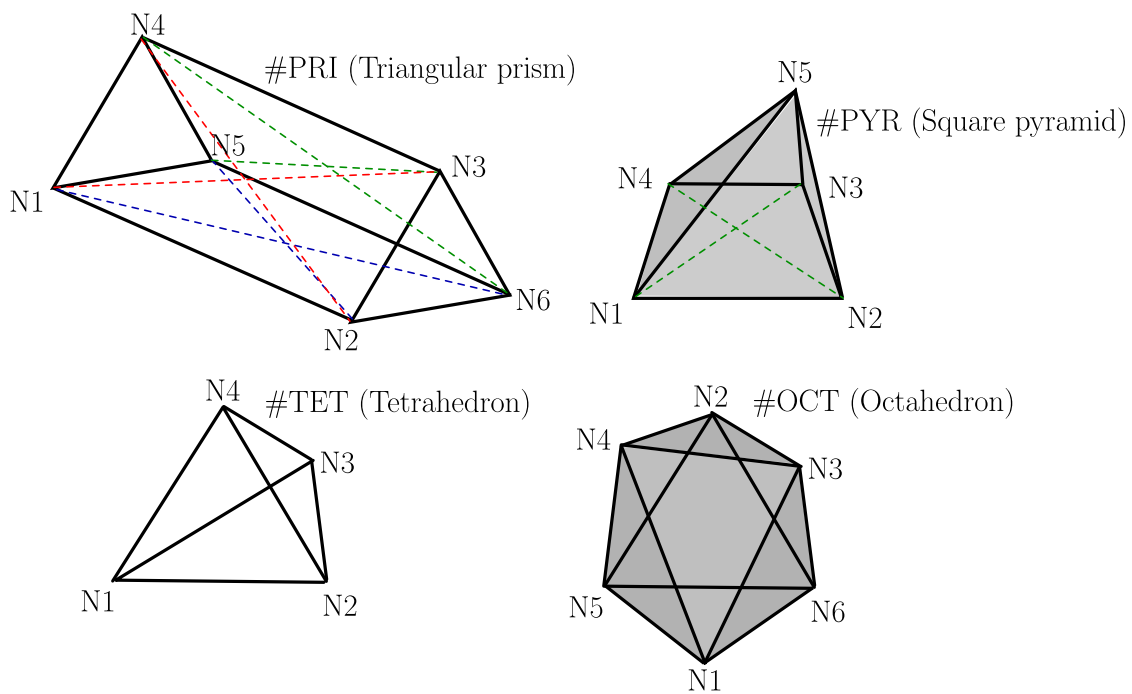

Fig. 5. Polyhedra generated by subdivision of a tetrahedron.

loading/unloading conditions in terms of stress:

$(1-d) \sigma_{\mathrm{eq}}-\bar{\sigma}(\bar{\varepsilon}) \leq 0$

$\dot{d} \geq 0$

$\dot{d}\left[(1-d) \sigma_{\mathrm{eq}}-\bar{\sigma}(\bar{\varepsilon})\right]=0$

from which a Hookean-like constitutive law is obtained, where Voigt notation is used:

$\mathbf{S}=(1-d) C \mathbf{E}$

and $C$ is the elastic tangent modulus. Function $\bar{\sigma}(\bar{\varepsilon})$ follows the standard exponential softening law:

$\bar{\sigma}(\bar{\varepsilon})=\sigma_{\max } \exp \left[-\frac{\bar{\varepsilon} L \sigma_{\max }}{G_{c}}\right]$

In (18), $\sigma_{\max }$ is the maximum equivalent stress, $G_{c}$ is the critical strain energy release rate (all modes are equally combined in $\varepsilon_{\text {eq }}$ ) and $L$ is the length scale parameter. To ensure consistence with other models, we identify $d$ in (14-16) and (17) as the damage constitutive variable.

Considering the model described by (14)-(18), two main approaches can be used:

1. Use of a cohesive law to introduce the dissipation of fracture energy in a process region which is explicitly a surface. This has been performed with technique such as XFEM [6] and alternatives.

2. Use of a regularized formulation, cf. [21,3], which provides an explicit length scale and avoids the cohesive elements.

The first approach is theoretically sound but makes use of an explicit crack path determination technique which can be difficult to find in 3D. Aspects such as crack coalescence and bifurcation are difficult to obtain in a robust form in 3D. The second approach, although much simpler since it does not require an explicit crack path, often produces unrealistic crack regions and also is difficult to fine-tune in terms of energy dissipation.

Remeshing is a form to remove the excessive thickness created by the regularized formulation and to ensure that dissipation is limited to a realistic region, without requiring crack path determination.

Implementation details, including the control equation for the solution, are provided in previous papers, see e.g. [1].

\section{Regularization with the screened Poisson equation}

The classical screened Poisson equation [7] (typically named
Helmholtz-like, cf. [21,3]) is adopted to regularize the otherwise illposed equilibrium problem in the presence of strain softening [5]. The combination with remeshing is very useful, since the usual irregular results produced by. Using an additional field, $\bar{\varepsilon}$, we perform a coupling with the constitutive-based $\varepsilon$ as:

$l\left(d, L_{m}\right)^{2}\left(\nabla_{b}^{2} \bar{\varepsilon}\right): \boldsymbol{I}=\bar{\varepsilon}-\varepsilon$

with the following boundary condition:

$\nabla_{b} \bar{\varepsilon} \cdot v=0 \quad$ in $\Gamma_{b}$

A version with constant $l$ was established by Lasry and Belytschko [16] who used an explicit version of this model, requiring higher-order continuity. In (19), $\nabla_{b}^{2}$ is the Laplacian with respect to the coordinates in equilibrium configuration $\Omega_{b}$, corresponding to an updatedLagrangian formulation. This allows the diffusion effect of (19) without the well-known [3] flattening side-effect. It is worth noting that $\bar{\varepsilon}$ given by equation ((19) is a weighted average (see [10,21]) and therefore mesh size only affects the local quantity $\varepsilon$ which is not directly responsible for softening, as $d$ is made dependent on $\bar{\varepsilon}$. In the present work we introduce the following function $l\left(d, L_{\mathrm{m}}\right)$ :

$l\left(d, L_{\mathrm{m}}\right)=L-d L_{\mathrm{m}}$

where $L_{\mathrm{m}}$ is the average mesh edge size at the localization region. The following constraint applies:

$L_{\mathrm{m}} \leq L$

this limits the spreading of the damaged region which occurs with fixed $l$. An alternative approach with similar effect was used by Geers et al. [10] by means of a rate effect. We use a staggered scheme to regularize the strain-softening problem. We introduce an element that implements equation (19) using the following weak form with the previous rate notation:

$\dot{W}_{\varepsilon}=\int_{\Omega_{b}}\left[-\left(L-d L_{\mathrm{m}}\right)^{2}\left(\nabla_{b} \bar{\varepsilon} \cdot \nabla_{b} \dot{\bar{\varepsilon}}\right)+(\varepsilon-\bar{\varepsilon}) \dot{\bar{\varepsilon}}\right] \mathrm{d} \Omega_{b}$

where, in terms of discretization for a tet, $\bar{\varepsilon}=\sum_{K=1}^{4} N_{K}(\xi) \bar{\varepsilon}_{K}$ where $N_{K}(\boldsymbol{\xi})$ are the classical shape functions and $\bar{\varepsilon}_{K}$ are the nodal unknowns for the regularization element. Linearization of (21) follows:

$\mathrm{d} \dot{W}_{\varepsilon}=\int_{\Omega_{b}}\left[-\left(L-d L_{\mathrm{m}}\right)^{2}\left(\nabla_{b} \mathrm{~d} \bar{\varepsilon} \cdot \nabla_{b} \dot{\bar{\varepsilon}}\right)-\mathrm{d} \bar{\varepsilon} \dot{\bar{\varepsilon}}\right] \mathrm{d} \Omega_{b}$

The implementation is performed with Mathematica [23] and AceGen software [15]. We obtain the following element residual for node $M$ : 


$$
h=0.2 \quad h=0.1
$$

Initial mesh
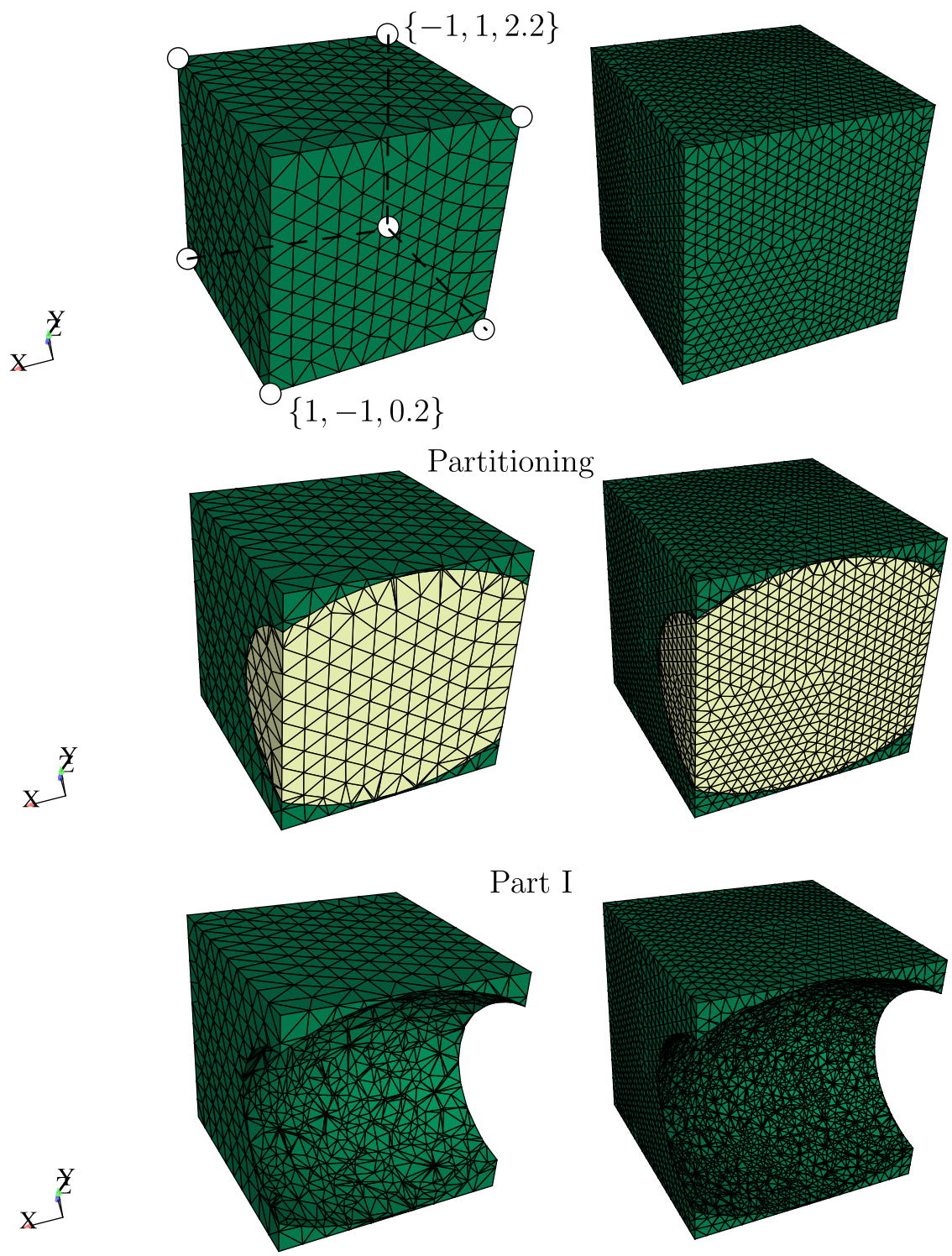

Ellipsoide: $\phi=\frac{x^{2}}{1.5^{2}}+\frac{y^{2}}{1^{2}}+\frac{z^{2}}{1.25^{2}}-1=0$

Fig. 6. Cube with intersecting ellipsoid: two distinct mesh densities.

$r_{M}^{e}=\int_{\Omega_{b}}\left[-\left(L-d L_{\mathrm{m}}\right)^{2} \frac{\partial N_{K}}{\partial x_{i}} \frac{\partial N_{M}}{\partial x_{i}} \bar{\varepsilon}_{K}+\left(\varepsilon-N_{K} \bar{\varepsilon}_{K}\right) N_{M}\right] \mathrm{d} \Omega_{b}=0$

where the sum symbol for $K$ was omitted. In (23), $N_{K}$ are the shape functions. The linearization corresponding to (23) provides the element stiffness matrix for nodes $M$ and $K$ as:

$K_{M K}^{e}=\int_{\Omega_{b}}\left[-\left(L-d L_{\mathrm{m}}\right)^{2} \frac{\partial N_{K}}{\partial x_{i}} \frac{\partial N_{M}}{\partial x_{i}}-N_{K} N_{M}\right] \mathrm{d} \Omega_{b}$

To compare this new technique with the conventional fixed- $l$ algorithm, we compare the localization regions in tension for a stretched bar in Fig. 8 where all relevant data is shown. We can observe that our new technique with variable $l$ results in a much sharper definition of the localization region.

\section{Numerical tests}

\subsection{Verification test: pulling exercise}

When performing full remeshing, mapping (or transference) of degrees-of-freedom and constitutive variables is required between the evolving meshes. In the presence of smoothing, this is also required. However, the mesh partitioning algorithm does not require that step. To assess the smoothness of results, as well as the effect of the updating parameter $f_{m}$ we introduce the following benchmark: using Kirchhoff/ Saint-Venant a bar is pulled up to double its original length. Fig. 9 shows the relevant data for this problem.

Differing from classical full remeshing and map algorithms, the reaction is very smooth since variable transfer is not required, see Fig. 10a. Mesh quality decreases with refinement, which is acknowl- 


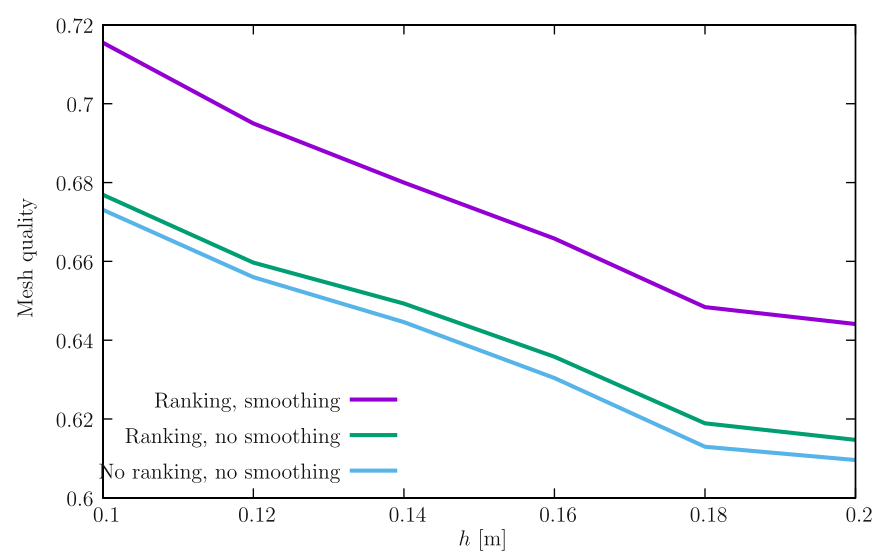

Fig. 7. Partition by an ellipsoid inside: comparison of strategies. Quality as a function of mesh characteristic length $h$.

edged in the literature (see $[25,4]$ ), as Fig. 10b shows. The parameter $f_{m}$ has a dramatic effect in the computational cost. For $h=1.5$ and $f_{m}=1.5$ the final number of elements is nearly eight times the initial, see Fig. 10c.

\subsection{Pristine three-point bending fracture}

We now consider a three-dimensional fracture problem, the threepoint bending beam, which is described in Fig. 11. In this Figure, consistent units are used for dimensions. In contrast with classical benchmarks, this does not include a pre-notch and is pristine in terms of initial damage. Fig. 11 shows the relevant data for this problem and the coarse initial mesh. The goal here is to assess the effect of $h_{\min }$ in the crack path and load/deflection results. Fig. 12a shows the reaction as a function of $\bar{v}$. Some conclusions can be drawn: without remeshing, results are excessively ductile. In addition, some sensitivity exists in terms of $h_{\min }$, which indicates the effect of poor-quality elements in the process region. This is further confirmed in Fig. 12b where a smaller $h_{\min }$ corresponds to a sharper decrease in mesh quality.

\subsection{Crack propagation and slanting in a knee-lever}

We now use the geometry and relevant data from Schöllmann et al. [26] for their knee-lever problem. The knee-lever is built from aluminum alloy IADS 7075T 651. Dimensions and constitutive properties are shown in Fig. 13a. Fracture properties agree with what was reported for this material in [26] and a simple proportional loading is performed here, with monotonously increasing $\bar{u}$. Since the thickness is moderate with respect to the other dimensions, some plane stress effect is noticeable in the crack advance, as Fig. 13b shows, with the wellknown slanting phenomenon. For the meshes with characteristic lengths $h=4$ and $h=5$, a sequence of deformed configurations is shown in Fig. 14. We point out that some roughness appears during propagation, in contrast with alternative methods by the Authors (see, e.g. [2]). In terms of mesh quality evolution, with the presence of smoothing we counteracted part of the quality-decreasing effect, as Fig. 15a shows. In contrast with the previous test, the process region is relatively localized and therefore a limited increase in the number of elements is observed, see Fig. 15a.

\section{Conclusions}

In the context of tetrahedral mesh cutting and subdivision, we introduced a pre-ordering in new mid-edge nodes to avoid the presence of non-tetrahedrizable prisms. We tested the algorithm in mesh partitioning, large strain analysis and computational fracture. In the latter,we combined a staggered algorithm with the modified screened Poisson equation and adaptive mesh refinement, and successfully solved two problems in quasi-brittle fracture. The Mazars equivalent strain definition [17] was used. Two main goals were achieved:

1. Crack path criteria are not required. Mesh refinement defines the crack path. The crack slanting phenomenon in plane stress is naturally reproduced.

2. Cohesive laws are not required. A regularized continuum law, within a smeared model, is used which provides the necessary dissipation (which is combined with the screened Poisson equation).

Besides the usual mesh length $h$, which is required to solve any finite element problem, only two additional solution parameters are required: the non-local length $L$ appearing in the screened-Poisson equation and also in the smeared model, and the mesh refinement length $h_{\min }$, controlling the subdivision of elements. When compared with enrichment methods or classical remeshing algorithms, we detected the following two shortcomings in the present technique:

1. Computational cost is comparatively high, since two analysis are performed, with the second involving regularization and equilibrium equations. Degrees-of-freedom also increase in the first stage of the analysis.

2. Crack paths are not smooth and present oscillations.

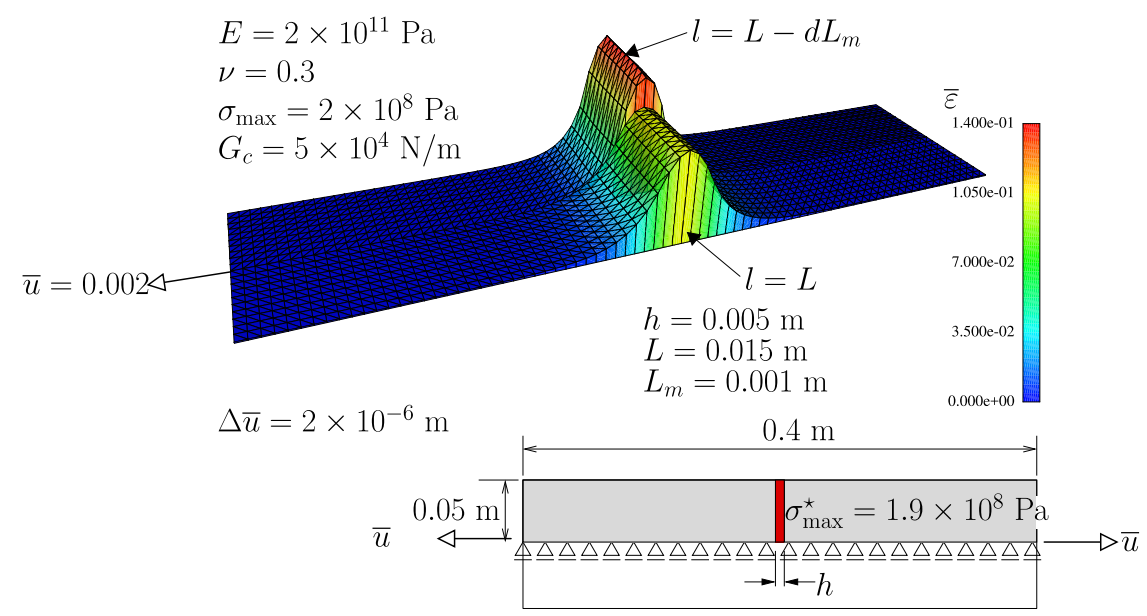

Fig. 8. Stretched bar under tension: localization region as a function of $\bar{\varepsilon}$ for $l=L$ and $l=L-d L_{m}$. 

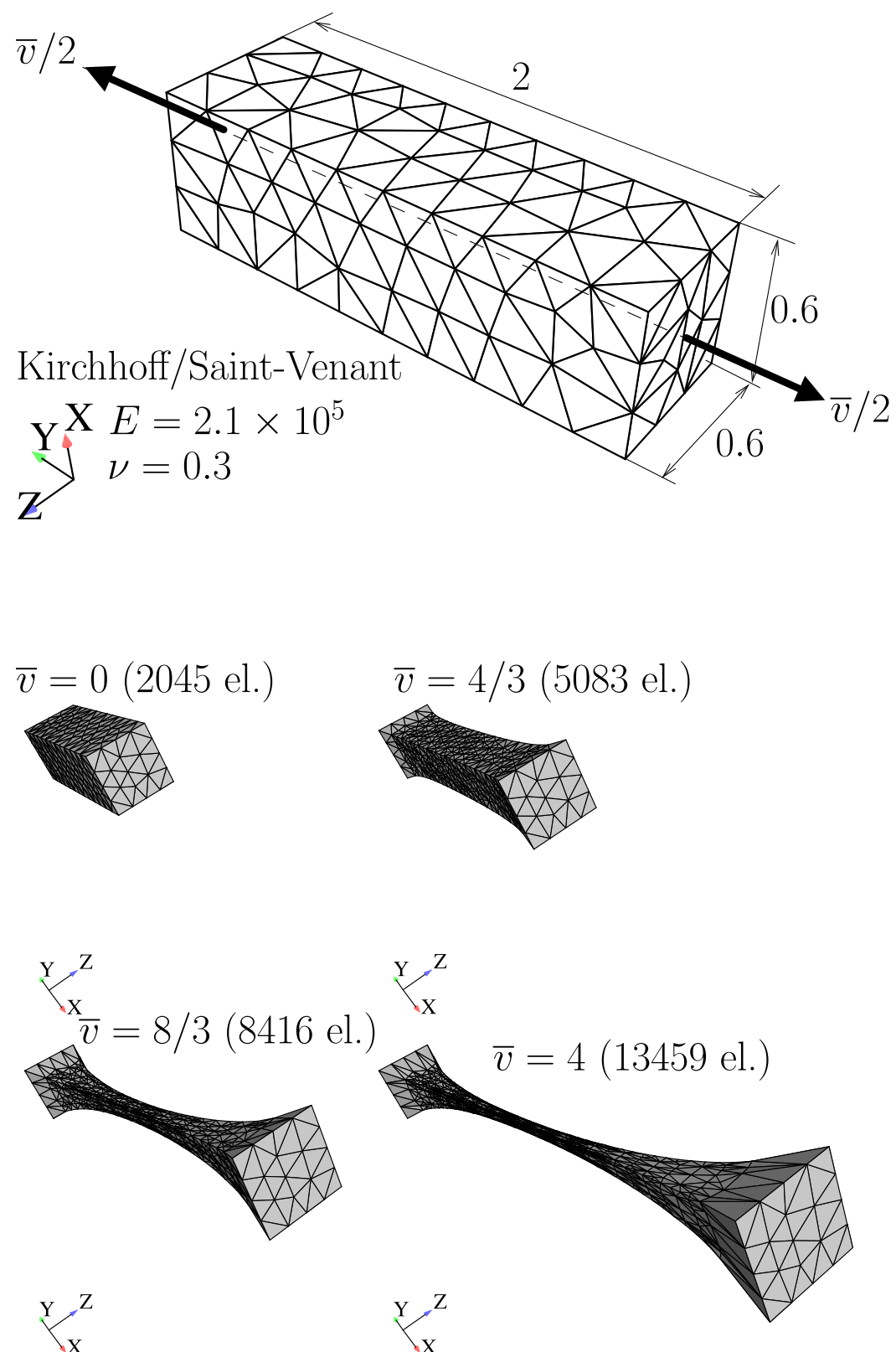

Fig. 9. Pulling exercise: mesh evolution for $f_{m}=1.5$. 


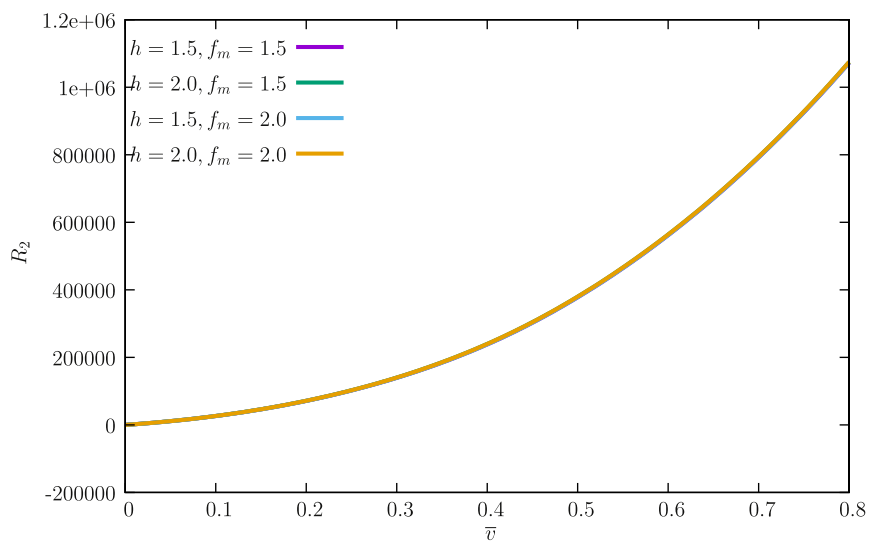

(a) Longitudinal reaction vs $\bar{v}$

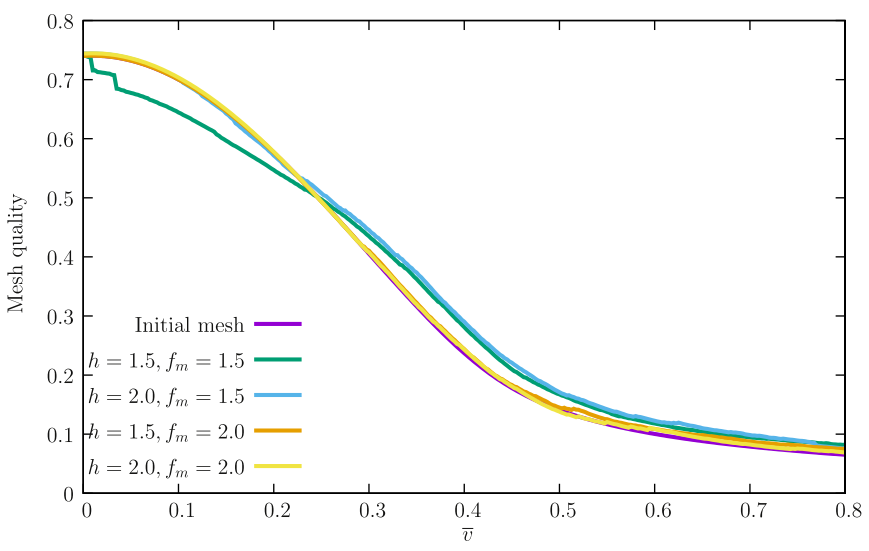

(b) Mesh quality vs $\bar{v}$

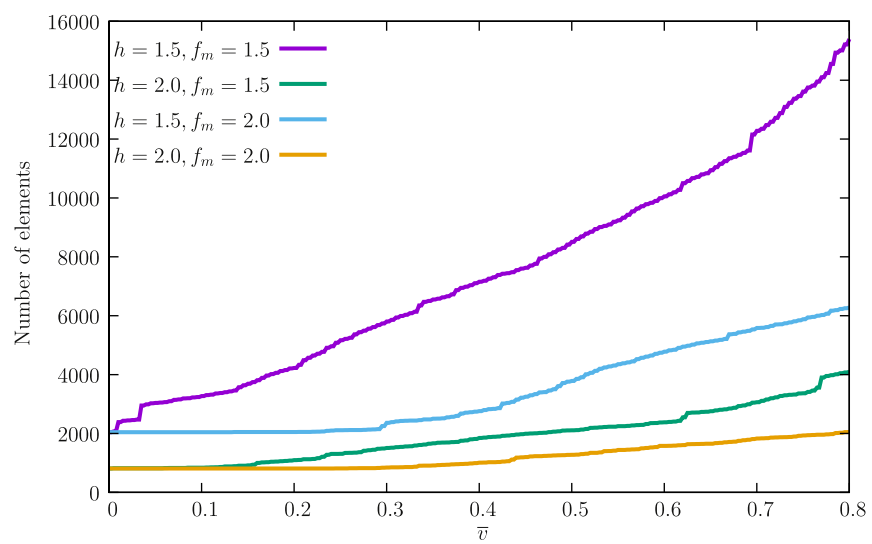

(c) Evolution of the number of elements as function of $\bar{v}$

Fig. 10. Evolution of reaction, mesh quality and number of elements as a function of $\bar{v}$. 

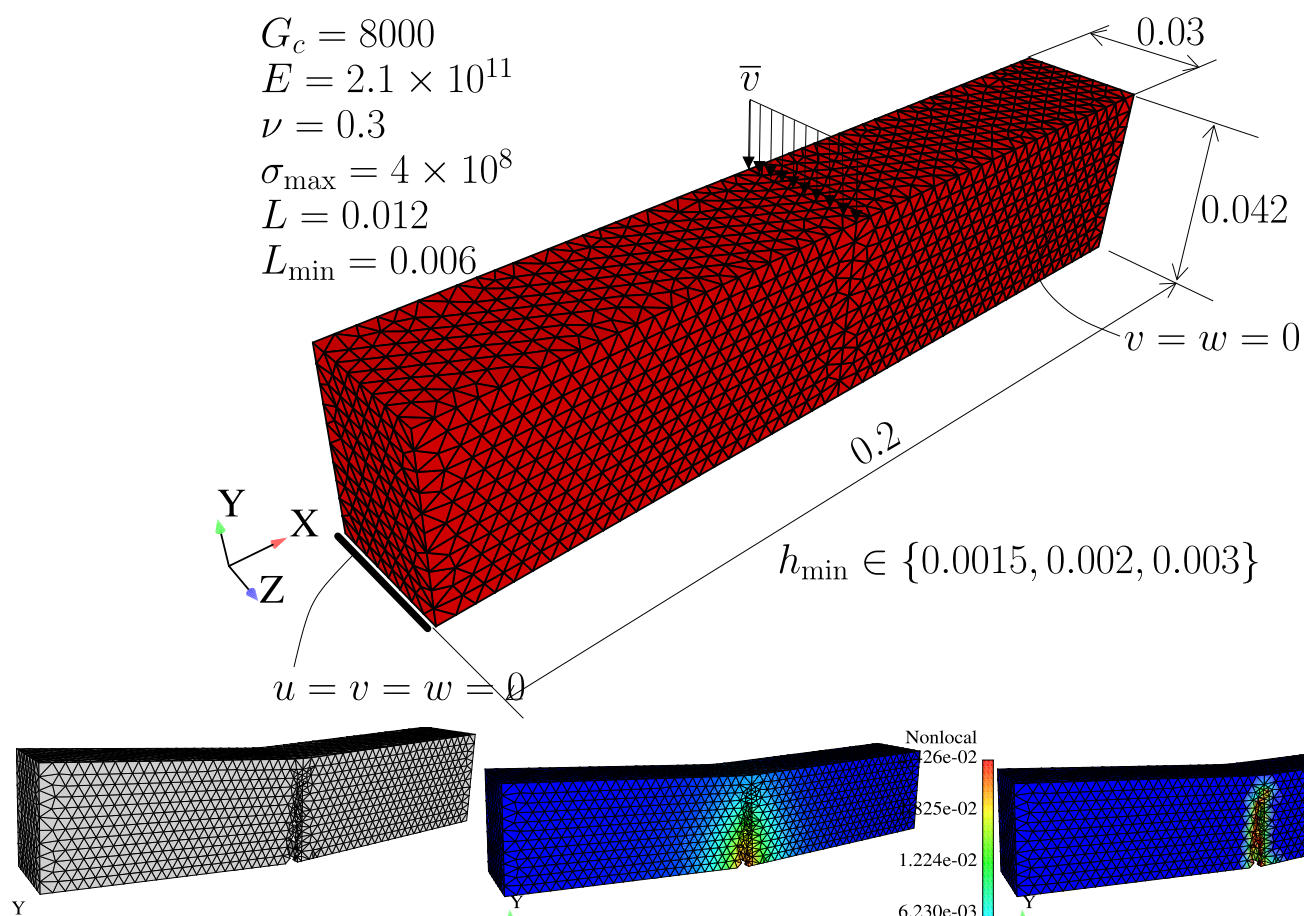

$\stackrel{\mathrm{Y}}{\mathrm{Z}} \mathrm{X} \quad h_{\min }=0.003$
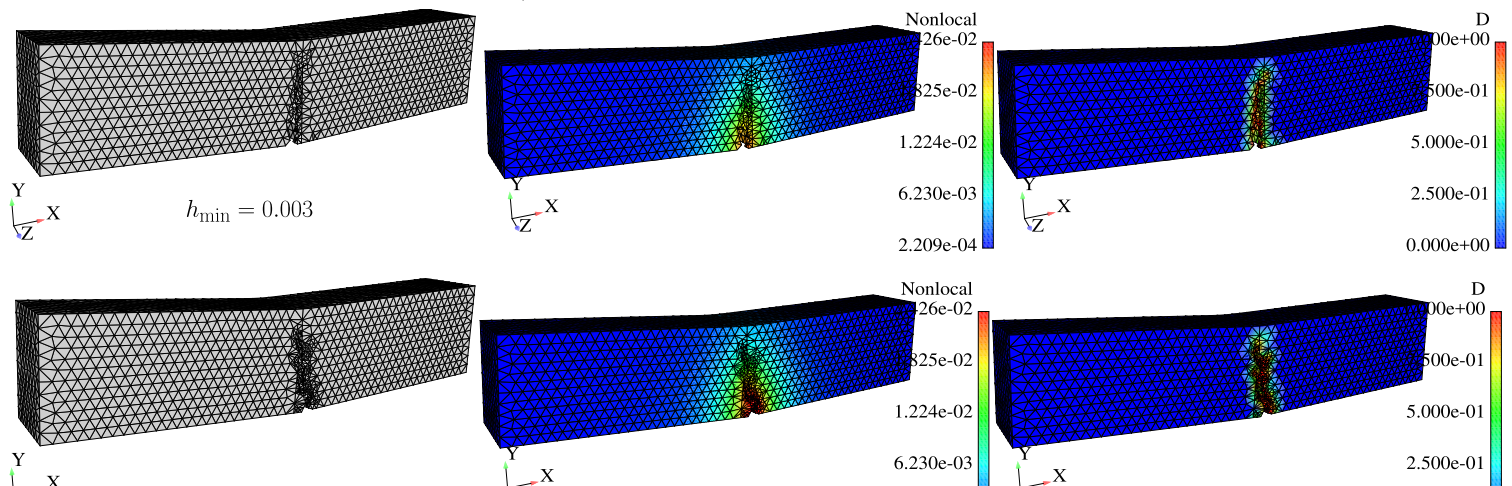

${ }_{Z} \mathrm{x}$

$h_{\min }=0.002$
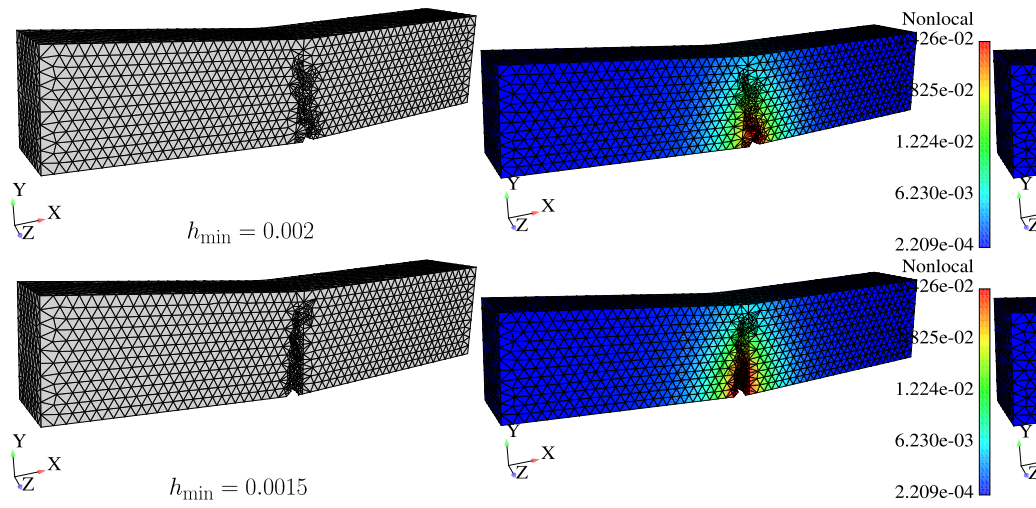

Fig. 11. Three-point bending test. 


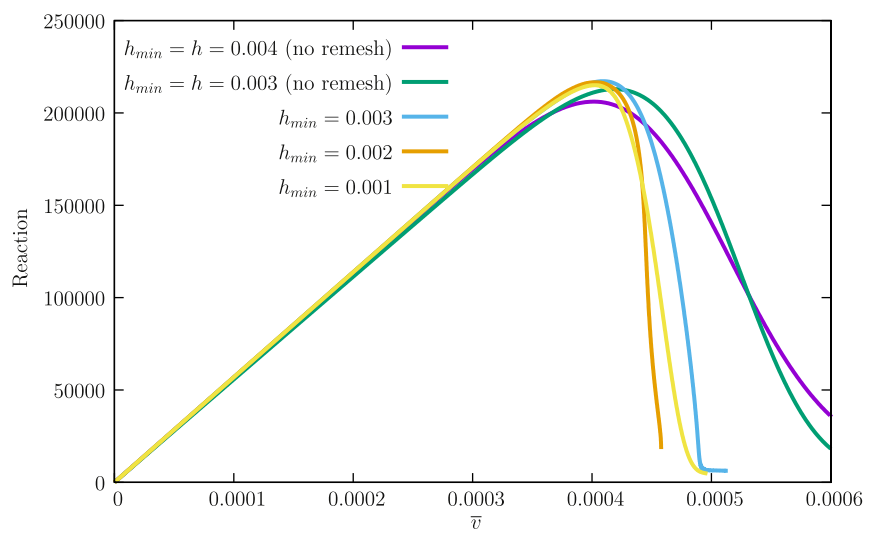

(a) Transversal reaction vs $\bar{v}$

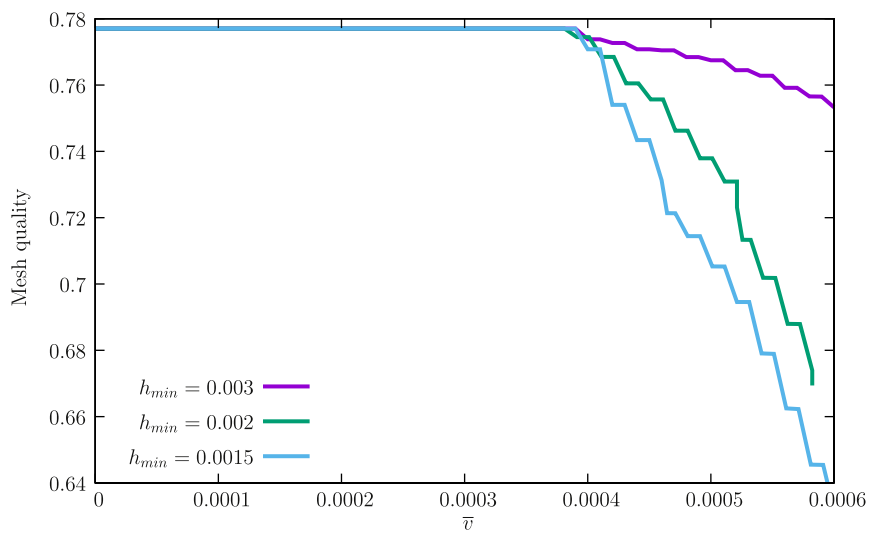

(b) Mesh quality as a function of $\bar{v}$

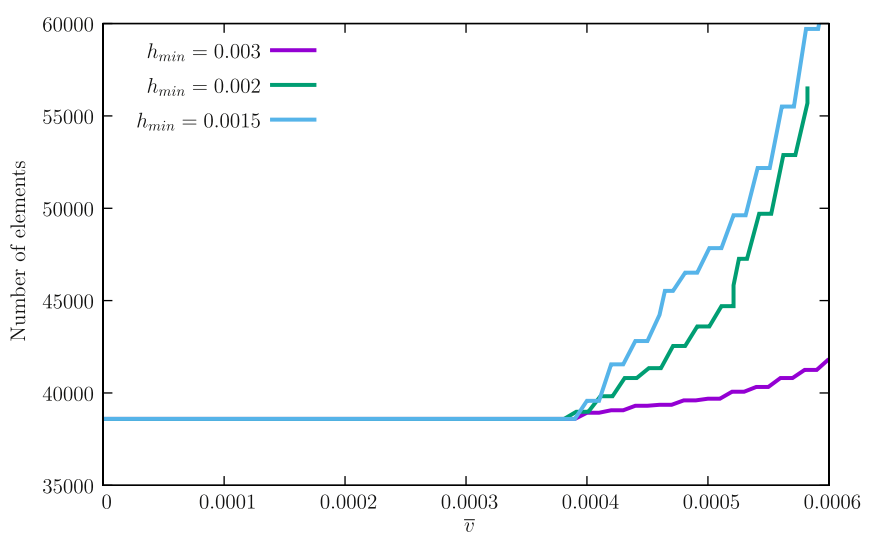

(c) Evolution of the number of elements as function of $\bar{v}$

Fig. 12. Three-point bending test: effect of $h_{\min }$. 


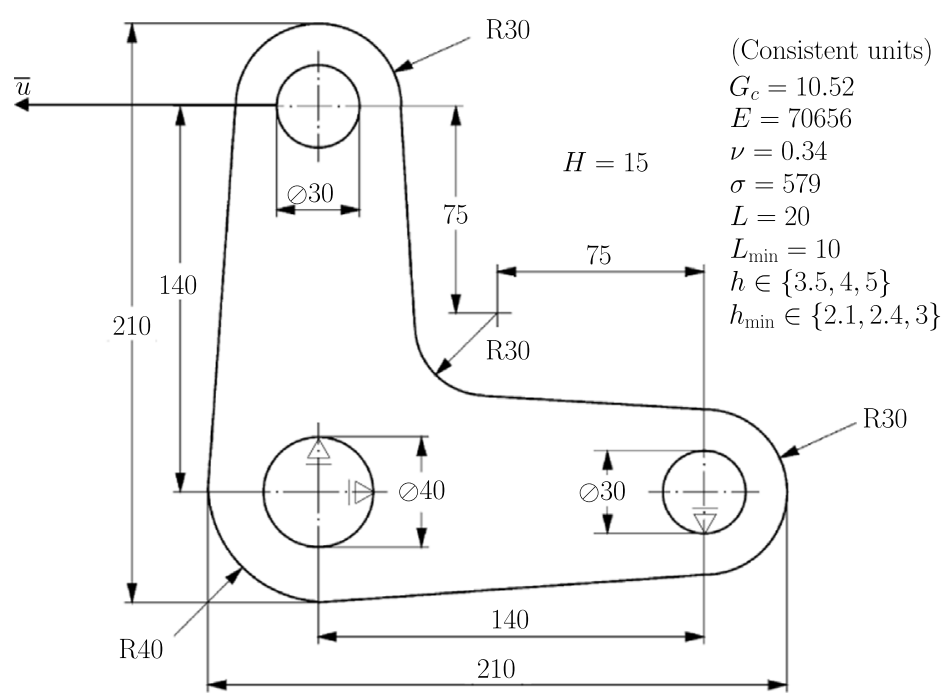

(a) Knee-lever: relevant dimensions and mechanical properties

Front view (slanted crack path)
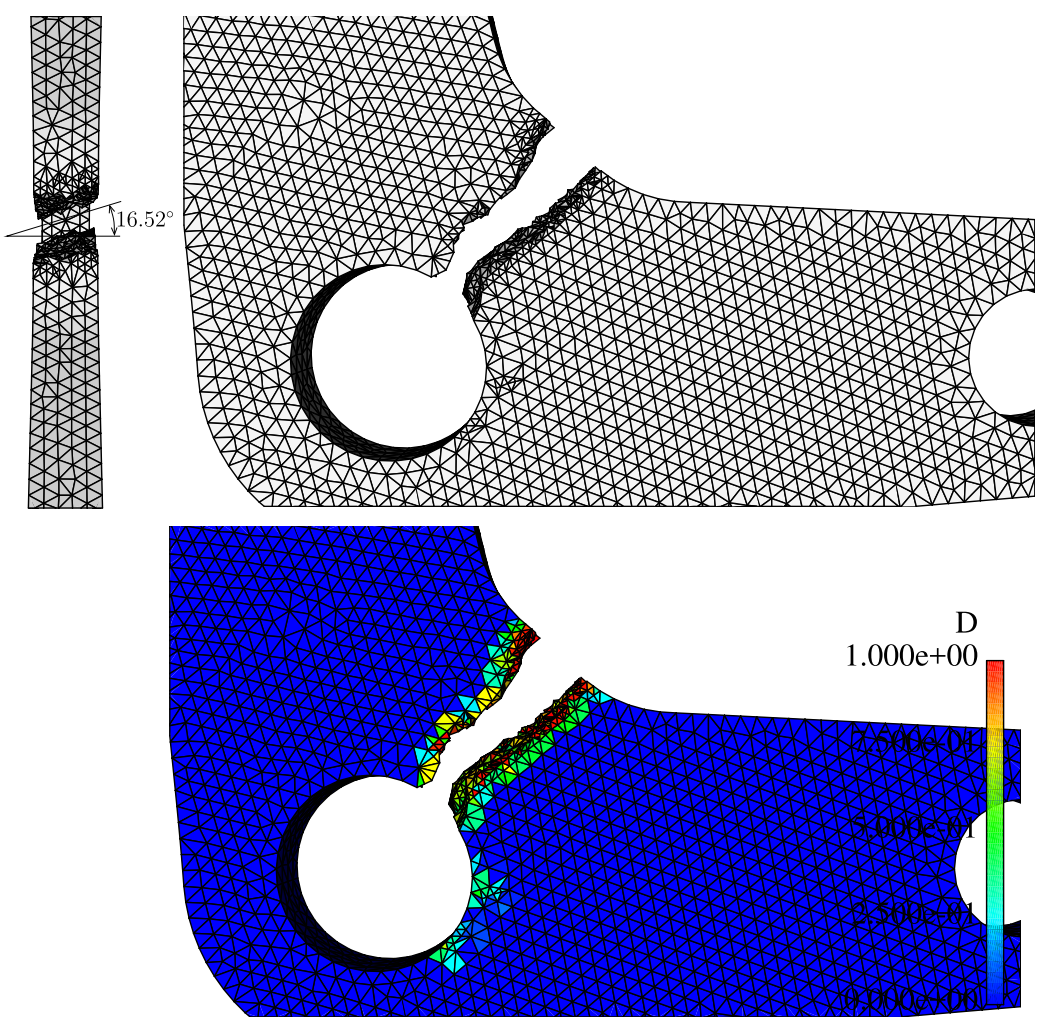

(b) Slanting for $h=3.5$.

Fig. 13. Knee-lever: geometry, relevant data and mesh evolution results. 

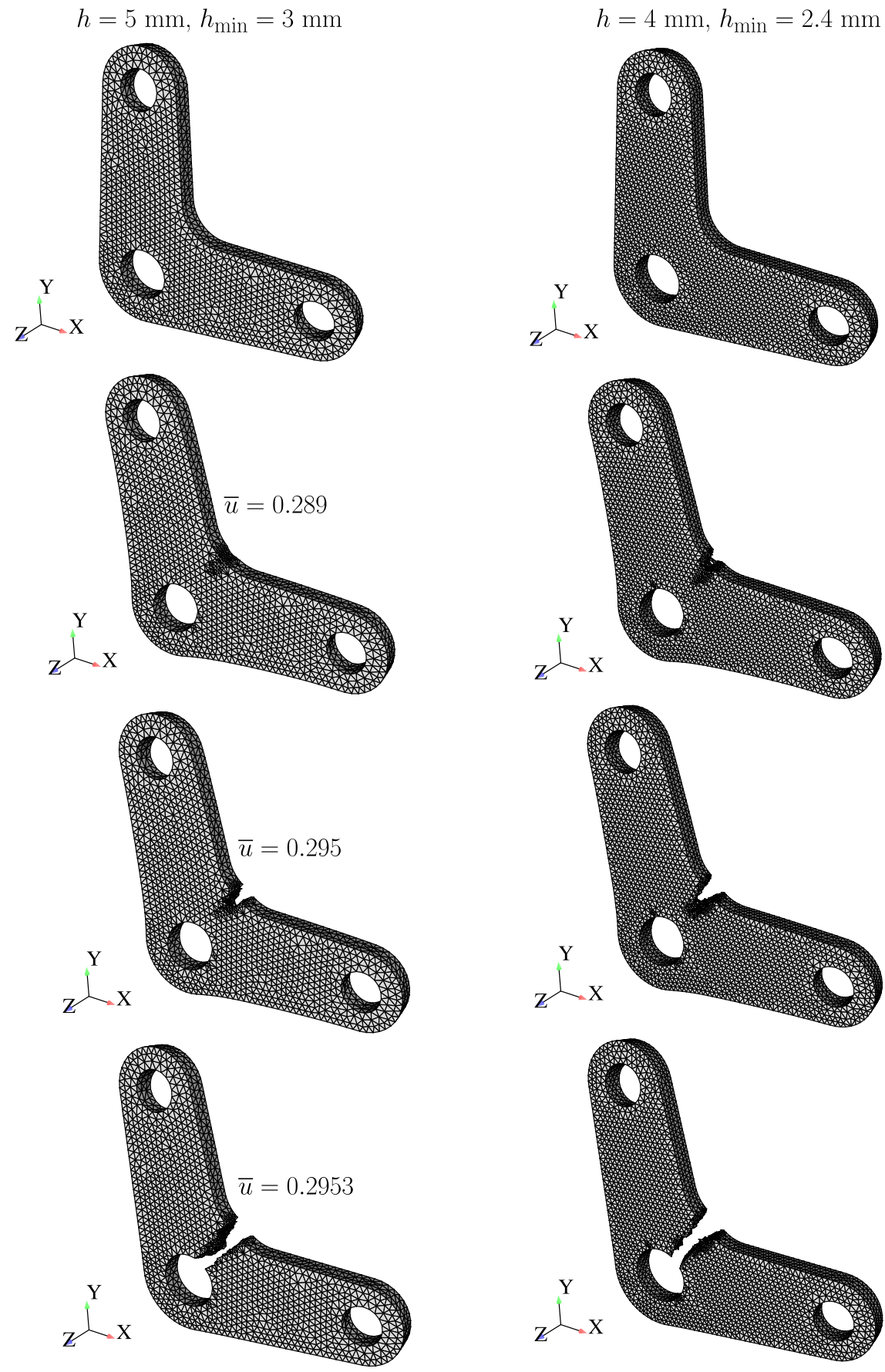

(10× magnified displacement)

Fig. 14. Knee-lever: sequence of deformed meshes for $h=4$ and $h=3 \mathrm{~mm}$. 


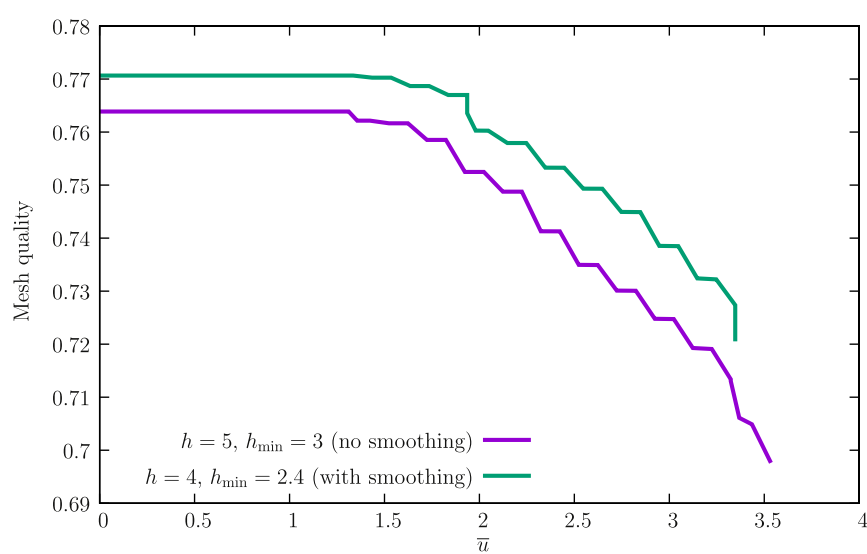

(a) Mesh quality vs $\bar{u}$

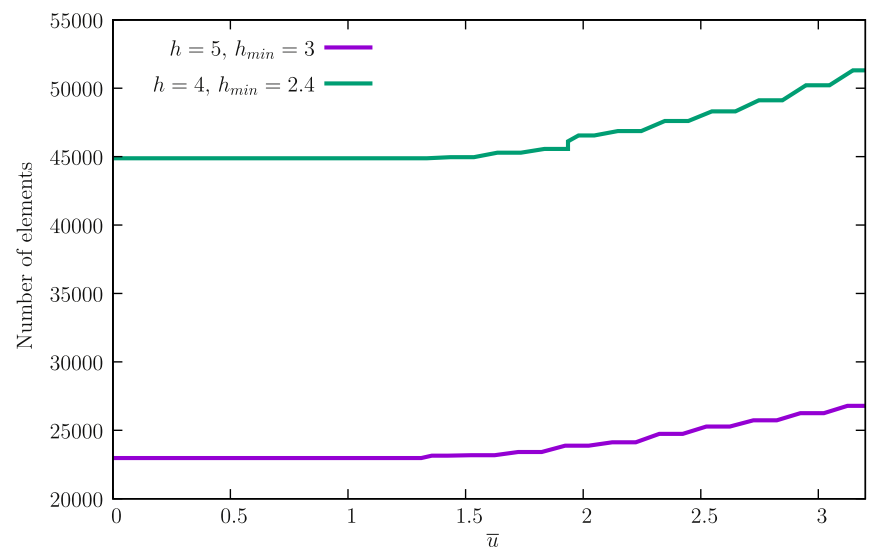

(b) Evolution of the number of elements as function of $\bar{u}$

Fig. 15. Knee-lever test: effect of $h$ and $h_{\min }$.

\section{References}

[1] P. Areias, J. Dias-da-Costa, Alfaiate, E. Júlio, Arbitrary bi-dimensional finite strain cohesive crack propagation, Comput. Mech. 45 (1) (2009) 61-75.

[2] P. Areias, T. Belytschko, Analysis of three-dimensional crack initiation and propagation using the extended finite element method, Int. J. Numer. Methods Eng. 63 (2005) 760-788.

[3] P. Areias, J.M.A. César de Sá, C.A. Conceição António, A gradient model for finite strain elastoplasticity coupled with damage, Finite Elem. Anal. Des. 39 (2003) 1191-1235.

[4] R. Bridson, J. Teran, N. Molino, R. Fedkiw, Adaptive physics based tetrahedral mesh generation using level sets, Eng. Comput. (Lond. 21 (2005) 2-18.

[5] R. de Borst, J. Pamin, M.G.D. Geers, On coupled gradient-dependent plasticity and damage theories with a view to localization analysis, Eur. J. Mech. A/Solids 18 (1999) 939-962.

[6] N. Moës, J. Dolbow, T. Belytschko, A finite element method for crack growth without remeshing, Int. J. Numer. Methods Eng. 46 (1999) 131-150.

[7] A.L. Fetter, J.D. Walecka, Theoretical Mechanics of Particles and Continua, Courier Dover (2003), 2003.

[8] P.J. Frey, P.-L. George, Mesh Generation: Application To Finite Elements, Hermes Science, Oxford, 2000.

[9] P. Fürnstahl, Consistent mesh partitioning using tetrahedral meshes (Master's thesis), Institute for Computer Graphics and Vision, Graz University of Technology, Inffeldgasse, 16. A-8010 Graz, Austria, 2005.

[10] M.G.D. Geers, R. de Borst, W.A.M. Brekelmans, R.H.J. Peerlings, Strain-based transient-gradient damage model for failure analysis, Comp. Method Appl. Methods Eng. 160 (1998) 133-153.

[11] P.-L. George, H. Borouchaki, Delaunay Triangulation and Meshing: application to Finite Elements, Hermes, Paris, 1998.

[12] P.L. George, Automatic Mesh Generation, John Wiley and Sons, 1991.

[13] G. Greiner, R. Grosso, Hierarchical tetrahedral-octahedral subdivision for volume visualization, Vis. Comput. 16 (6) (2000) 357-369.

[14] H. Huang, L. Xue, Prediction of slant ductile fracture using damage plasticity theory, Int J Pres. Ves. Pip. 86 (2009) 319-328.

[15] J. Korelc, Multi-language and multi-environment generation of nonlinear finite element codes, Eng. Comput. 18 (4) (2002) 312-327.

[16] D. Lasry, T. Belytschko, Localization limiters in transient problems, Int. J. Solids Struct. 24 (1988) 581-597.

[17] J. Mazars, Application de la mécanique de l'endommagement au comportement non linéaire et à la rupture du béton de structure. Thèse de Doctorat d'Etat, Université Paris VI, Paris, France, 1984.

[18] H.-W. Nienhuys, F.A. van der Stappen, A Surgery Simulation Supporting Cuts and Finite Element Deformation, pages 145-152. Springer Berlin Heidelberg, Berlin, Heidelberg, 2001.

[19] R.W. Ogden, Non-linear elastic deformations, Dover Publications, Mineola, New York, 1997.

[20] C.J. Paulus, L. Untereiner, H. Courtecuisse, S. Cotin, D. Cazier, Virtual cutting of deformable objects based on efficient topological operations, Vis. Comput. 31 (2015) 831-841.

[21] R.H.J. Peerlings, R. de Borst, W.A.M. Brekelmans, J.H.P. de Vree, Gradient enhanced damage for quasi-brittle materials, Int. J. Numer. Methods Eng. 39 (1996) 3391-3403.

[22] A. Plaza, G.F. Carey, Local refinement of simplicial grids based on the skeleton, Appl. Numer. Math. 32 (2000) 195-218.

[23] Wolfram Research Inc., Mathematica, 2007.

[24] L. Rodríguez, I. Navazo, A. Vinacua, Data-driven tetrahedral mesh subdivision, Comput. Graph. Forum 26 (4) (2007) 783-793.

[25] D. Ruprecht, H. Müller, A scheme for edge-based adaptive tetrahedron subdivision, in: H.-C. Hege, K. Polthier (Eds.), Mathematical Visualization: Algorithms, Applications and Numerics, Springer Berlin Heidelberg, Berlin, Heidelberg, 1998.

[26] M. Schöllmann, M. Fulland, H.A. Richard, Development of a new software for adaptive crack growth simulations in 3D structures, Eng. Fract. Mech. 70 (2003) $249-268$. 